\title{
Differential Expression of MiR-18b in PBMC from T1D Patients in Isfahan Population
}

\author{
Zahra Azhir ${ }^{1}$, Zohreh Hojati ${ }^{1^{*}}$ \\ ${ }^{1}$ Division of Genetics, Department of Biology, Faculty of Sciences, University of Isfahan, Isfahan, Iran.
}

Received: 13 Sep 2016

Revised : 8 Oct 2016

Accepted: 20 Oct 2016

Corresponding Author: Zohreh Hojati

Division of Genetics, Department of Biology, Faculty of Sciences,

University of Isfahan, Isfahan, Iran.

Phone: +983117932478

E-mail: z.hojati@sci.ui.ac.ir

\begin{abstract}
Background: Type 1 diabetes (T1D) is caused by cell-mediated autoimmune attack on pancreatic beta cells. Previous studies highlight the role of microRNAs (miRNAs) in the pathogenesis of T1D. MiRNAs are small non-coding RNAs involved in the regulation of gene expression post-transcriptionally. In this work, microRNA-18b (miR-18b) was chosen and its differential expression was measured between T1D patients and healthy controls from Isfahan population.

Materials and Methods: miR-18b was selected according to bioinformatic studies using miRWalk software. 22 T1D patients and 18 healthy controls from Isfahan population were enrolled in this study. Total RNA of the peripheral blood mononuclear cell (PBMC) samples was extracted. After cDNA synthesis, the expression profile of miR-18b was quantified by means of qPCR method in patients and controls. Finally, the results were statistically analyzed.

Results: In this study despite our hypothesis, the expression levels of miR-18b didn't show any significant difference between T1D patients and healthy controls ( $\mathrm{p}$ value: 0.145 ).
\end{abstract}

Conclusion: Due to the results of our experimental analysis, it seems that miR$18 \mathrm{~b}$ doesn't have any association with T1D disease in Isfahan population.

Keywords: microRNA, miR-18b, miRWalk, qPCR, Type 1 diabetes (T1D)

Please cite this article as: Azhir Z, Hojati Z. Differential Expression of MiR-18b in PBMC from T1D Patients in Isfahan Population. Res Mol Med. 2016; 4 (4): 45-50

\begin{abstract}
Introduction
Type 1 diabetes (T1D) results from the autoimmune destruction of pancreatic islet- $\beta$ cells which produce insulin (1). In this disease, genetics, epigenetics and environmental factors interact with each other and make it a multifactorial complex disease (2). T1D can occur at any ages, but it is known as one of the most common chronic diseases in children (3). The incidence of T1D has been shown to increase for decades and the number of new T1D cases is increasing at the rate of about 3\% per year in different countries worldwide. This increase indicates the importance of environmental factors (46). Genetic susceptibility plays a critical role in the pathogenesis of T1D, in which genome-wide association studies (GWAS) have linked more than 50 genetic variants to the T1D susceptibility $(6,7)$. The major susceptibility locus is related to HLA class II genes which are located on chr6p21. In addition,
\end{abstract}

multiple non-HLA loci play effective role in the T1D risk, including INS, PTPN22, CTLA4, IL2RA, etc. (8-10). The CTLA4 (cytotoxic $\mathrm{T}$ lymphocyteassociated protein 4) gene participates in the regulation of $\mathrm{T}$ cell activation. This gene is a potential candidate for autoimmune diseases like T1D (10). Different types of T cells have a vital role in the pathogenesis of T1D (11). Among them, Th17 cells (a subpopulation of $\mathrm{CD} 4+\mathrm{T}$ cells) are considerable. Several reports highlight the role of the plasticity of Th17 cells and the balance between them and $T$ regulatory cells or Tregs (inhibitors of immune responses), in the pathogenesis of various autoimmune diseases including T1D $(1,12)$. miRNAs are 20-22 nucleotide non-coding RNAs regulating gene expression after translation (13). These molecules are involved in various physiological processes, including cell proliferation, cell differentiation, apoptosis, 
maturation and inflammation (14, 15). Many miRNAs have been reported to be expressed differently in T1D and may have a critical role in the pathogenesis of this disease and autoimmunity through the regulation of key genes. So these molecules can be used as useful biomarkers in the early detection and cure of T1D (16-19). For this purpose, bioinformatic databases were used in order to find an appropriate miRNA that could be related to T1D and finally miR-18b was chosen. In addition, miR-18b was previously reported to be upregulated in the PBMCs of T1D patients by microarray analysis (20). Here, the expression levels of miR-18b were quantified in the PBMCs of T1D patients from Isfahan population and compared to healthy controls by qPCR method. The expression levels of this miRNA didn't show any significant difference between T1D patients and healthy controls.

\section{Materials and methods}

The methods used in this study are summarized in Figure 1.

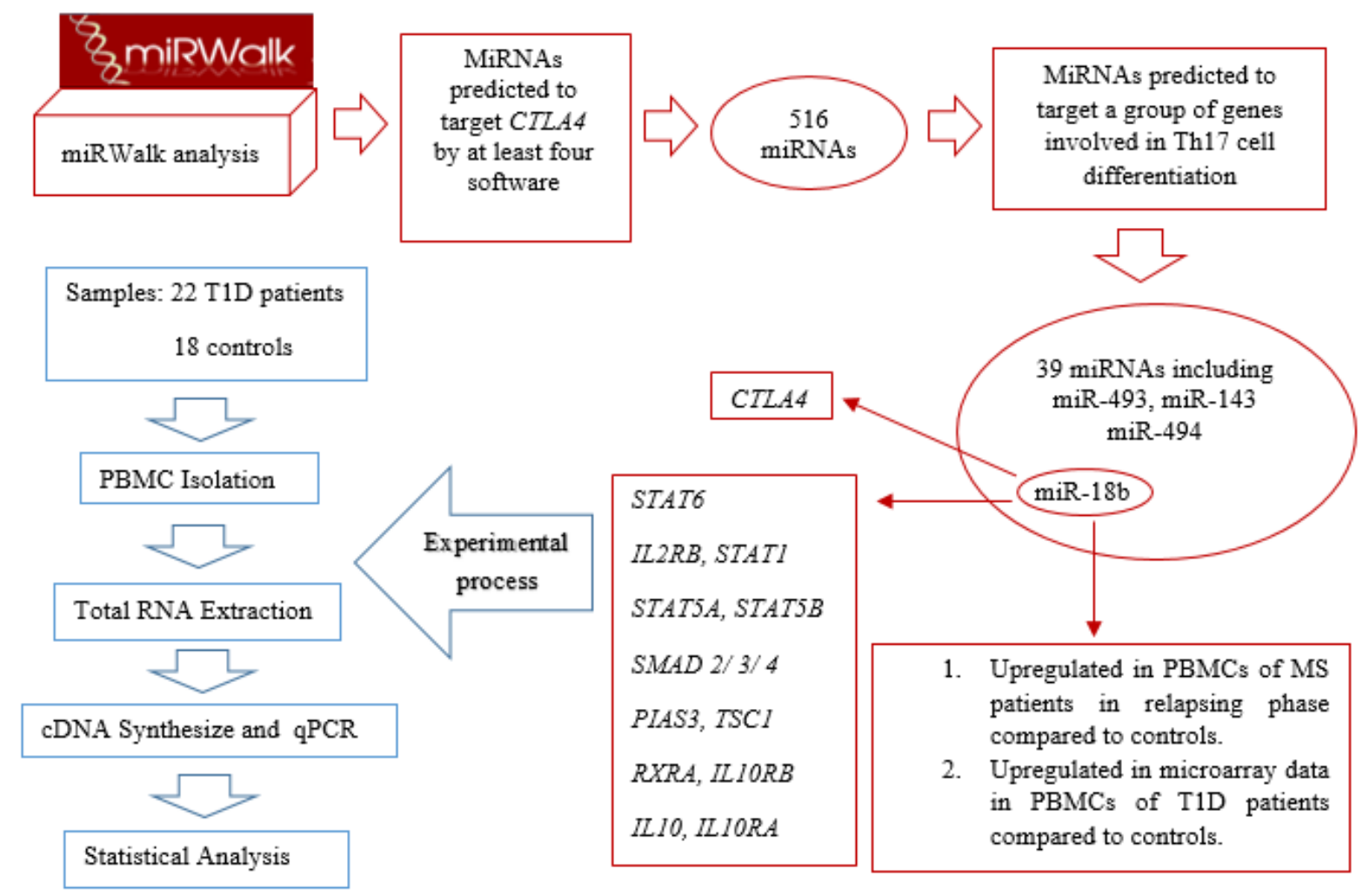

Figure 1. Workflow of the Bioinformatics and experimental processes in this study. Bioinformatics process indicated in red borders and experimental processes shown in blue borders.

\section{In silico studies}

In this study, miRWalk 2.0 software was used for prediction of miRNAs and only miRNAs which were predicted by at least four software were considered $(21,22)$. First, miRNAs predicted to target CTLA4 gene were noted, then a list of about 54 validated genes involved in Th17 cell differentiation was prepared and miRNAs which also targeted some of these genes were chosen from the previous list. The final list consisted of miRNAs which had differential expression in autoimmune diseases in addition to mentioned properties. Finally, miR-18b which was predicted to target CTLA4 gene and several key involved in Th17 cell differentiation was selected.
Sample collection and isolation of peripheral blood mononuclear cells (PBMCs)

An informed consent form was signed by all subjects enrolled in this study and was approved by the University of Isfahan Ethical Committee. The whole blood samples ( $5 \mathrm{ml}$ in EDTA containing tube) were drawn from 22 T1D patients and 18 healthy controls. All the subjects were between the ages of 13-30 years old and from Isfahan state population. The disease duration of patients was between 4-25 years. The patient group involved 13 females and 9 males and the control group consisted of 13 females and 5 males. The healthy controls had no family history of T1D and other autoimmune diseases. Briefly, the 
whole blood was diluted in 1:1 PBS and layered over a Hypaque-Ficoll (Innotrain, Germany) medium with half the volume of blood in a falcon tube. Fresh PBMCs were isolated using Hypaque-Ficoll density gradient centrifugation following phosphate buffered saline (PBS) washing.

\section{RNA extraction and cDNA synthesis}

Total RNA, including miRNAs, was extracted with Trizol reagent (Invitrogen, USA) from isolated PBMC samples according to manufacturer's instruction and afterwards the quantity and quality of extracted RNAs were verified by $1.5 \%$ agarose gel and 260/280 absorbance ratio. cDNA synthesis of $2 \mu \mathrm{g}$ isolated RNA was conducted for miR-18b and RNU6B using miRNAs complementary DNA (cDNA) Synthesis Kit (ParsGenome, Iran) based on manufacturer's instruction.

\section{Real time PCR ( $q P C R)$}

The $\mathrm{qPCR}$ reaction was carried out in a total volume of $15 \mu$ l, involved cDNA synthesized of $2 \mu \mathrm{g}$ RNA, added to a master mix containing $10 \mathrm{pmol} / \mu \mathrm{l}$ of miR$18 \mathrm{~b}$ specific primer (Parsgenome, Iran) and $7.5 \mu \mathrm{l}$ SYBR Green (TAKARA, Japan). The sequence of miR-18b is UAAGGUGCAUCUAGUGCAGUUAG (www.miRbase.org). The qPCR method was performed using Bio-Rad equipment with $95{ }^{\circ} \mathrm{C}$ for 5 min followed by 40 cycles of $95^{\circ} \mathrm{C}$ for $5 \mathrm{~s}, 63^{\circ} \mathrm{C}$ for $20 \mathrm{~s}$ and $72{ }^{\circ} \mathrm{C}$ for $30 \mathrm{~s}$. In this study miRNA expression was normalized by the endogenous control gene of small RNA, RNU6B (U6), as the reference gene (Figure 2).

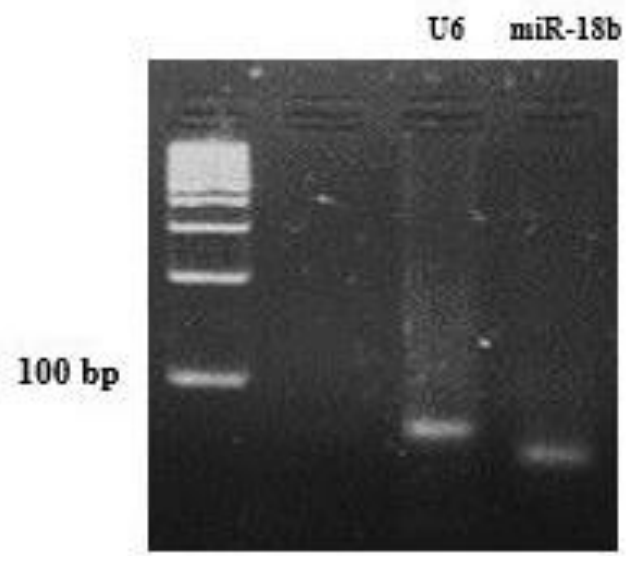

Figure 2 Visualization of miR-18b and RNU6B (U6) on agarose gel $4 \%$. Both miRNAs have a single band and are located beyond the $100 \mathrm{bp}$ band of ladder. Marker in here is a $100 \mathrm{bp}$ DNA ladder.

\section{Statistical analysis}

The REST (Relative Expression Software Tool) program was used for data analysis (23). Subsequently, nonparametric Mann-Whitney U test was performed in order to determine the significance or nonsignificance of variations between controls and patients using SPSS 16 software (SPSS Inc. 2007. Chicago) and GraphPad Prism 6 (GraphPad prism Software, Inc. San Diego CA, USA). The expression levels of this miRNA were also analyzed in association with gender, disease duration, the age at disease onset and $\mathrm{HbAlc}$ levels. As mentioned before, U6 gene which was confirmed as an appropriate reference gene in previous studies, was used in order to normalize final data $(17,19)$. P value $<0.05$ was considered statistically significant.

\section{Results}

In this study, 516 miRNAs were predicted to target CTLA4 gene by at least four software using miRWalk 2.0. Among these, 39 miRNAs had differential expression in autoimmune diseases and were predicted to target some genes of our list which were involved in Th17 cell differentiation.

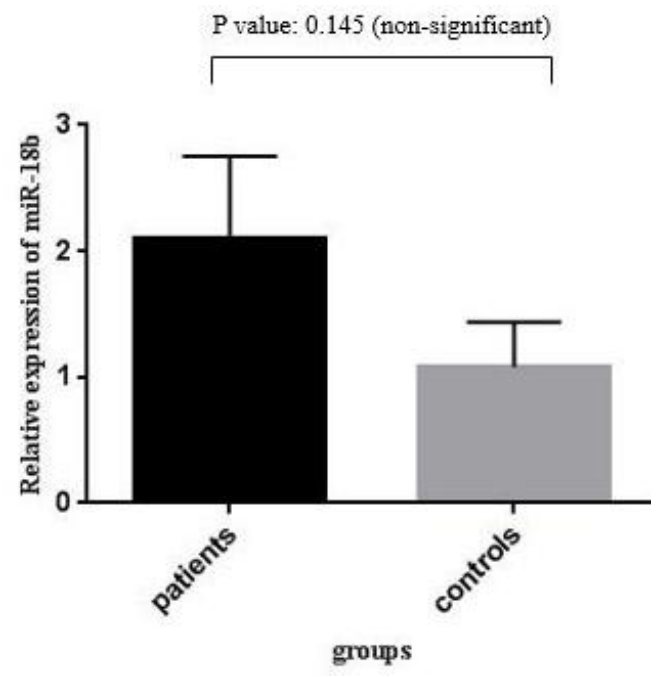

Error! Reference source not found.. The expression level of miR-18b in T1D patients (n: 22) compared to healthy controls (n: 18). Results normalized with RNU6B gene. $P$ value $<0.05$ was considered statistically significant. There is no significant difference between T1D patients and healthy controls ( $p$ value: 0.145 , non-parametric Mann-Whitney U test).

Finally, miR-18b was chosen as an appropriate candidate related to T1D. This miRNA was predicted to target STAT6, IL2RB, STAT1, STAT5A, STAT5B, SMAD2/3/4, PIAS3, TSC1, RXRA, IL10RB, IL10, IL10RA and FOXO3 genes in addition to CTLA4. The expression levels of miR$18 \mathrm{~b}$ and U6 (as reference gene) were measured by the qPCR method in the PBMCs of 22 patients and 18 healthy controls from Isfahan population. Next, data was normalized with U6 and the statistical analysis was performed by REST, SPSS and GraphPad Prism 6 software. The statistical results show no significant 
changes in miR-18b expression levels between patients and controls (p value: 0.145) (Figure 3). In addition, no significant changes were found in the expression levels of this miRNA in correlation with gender, disease duration, the age at disease onset and HbA1c levels.

\section{Discussion}

T1D is a complex multifactorial disease affecting millions of people around the world. There are at least 20 regions of the genome noted to link with the susceptibility to T1D (9). PTPN22, UBASH3A, and CTLA4 loci are mapped to have gene variants, which downregulate TCR activation response (2). CTLA4 gene is located on chromosome $2 \mathrm{q} 33$ and codes an important molecule for proper negative regulation of immune responses, which have reported severe lymphoproliferative disorders in knockout mice (24, 25). Treg cells expressing the transcription factor Foxp3 have a critical role in suppression of inflammation and maintenance of immunological self-tolerance. A specific deficiency of CTLA4 in Tregs of mice shows these cells may critically require CTLA4 in order to suppress immune responses (26, 27). The 3'-UTR rare variants in the CTLA4 gene may affect T1D pathogenesis by modification of miRNA-binding sites and thus gene regulation through miRNAs (28). The importance of environment in the pathogenesis of T1D highlights the role of epigenetic mechanisms which link the environment and gene function (29). Epigenetics involves mechanisms which cause stable and heritable changes in gene expression without alterations in DNA sequence, which can have an influence on autoimmunity development (30). Among epigenetic processes DNA methylation, histone modifications, chromatin accessibility and miRNAs can be noted (31). The differential expression of miRNAs can affect the particular targets and pathways which cause autoimmune diseases $(32,33)$. Previous studies show T1D is associated with differential expression of specific miRNAs in the blood and according to the role of these molecules in the immune homeostasis, they can be proper candidates for exploring the mechanism of pathogenesis, early detection and cure of T1D $(16,17,19)$. In this study, miRWalk was used as a bioinformatic tool for the prediction of appropriate miRNAs which target CTLA4 as an important gene in T1D susceptibility. hsa-miR-18b was selected as a proper candidate to target CTLA4 by screening $516 \mathrm{miRNAs}$ using at least four software. According to miRWalk results, miRWalk, miRMap, RNAhybrid and TargetScan software predicted CTLA4 as a target of miR-18b. This target was predicted for miR-18b by 90 percent confidence using TargetScan software. In addition, it is predicted that this miRNA targets, IL2RB, STAT1, STAT6, STAT5A, STAT5B, SMAD2/3/4, PIAS3, TSC1, RXRA, IL10RB, IL10, IL10RA and FOXO3 which all are negative regulators of Th17 cell differentiation. So, it has been predicted that miR-18b would be overexpressed in T1D and by inhibition of CTLA4, negative regulators of Th17 cell differentiation and therefore disruption of the balance between TReg/Th17 cells, may have a role in T1D pathogenesis. This miRNA is a member of miR-106a/363 cluster, which is located on chrX.q26.2 and expressed in CD4+ and CD8+ $\mathrm{T}$ cells $(34,35)$. Despite our prediction by bioinformatic tools, the expression levels of miR-18b didn't show any significant difference between T1D patients and healthy controls, so it seems there is no correlation between the expression levels of miR-18b and T1D in Isfahan population. However, our in silico analyses suggest mentioned genes as predicted targets of miR-18b which needs to be experimentally validated. The relative expression levels of miR-18b also seem not to be associated with gender, disease duration, the age at disease onset and HbAlc levels in Isfahan population. It was reported that $\mathrm{miR}-18 \mathrm{~b}$ is overexpressed in relapsing groups with multiple sclerosis compared to controls and may be associated with the relapse phase of multiple sclerosis (36). The miRNA expression profile of 11 T1D patients compared to 9 healthy controls by microarray slides showed that miR-18b is significantly upregulated in the PBMCs of T1D patients. In addition, their functional annotation studies showed this miRNA may be involved in various pathways, including apoptosis, chemokine signaling pathway, infection, focal adhesion, gap junction, hedge hug signaling pathway, insulin signaling pathway, MAPK signaling pathway, P53 signaling pathway, $\mathrm{T}$ cell receptor signaling pathway, TGF $\beta$ signaling pathway and Wnt signaling pathway. Furthermore HORMAD2, CLEC16A, ORMDL3, MTMR3 and LIF genes were predicted to be the targets of miR-18b (20). In the present study, we quantified the expression levels of miR-18b in the PBMCs of T1D patients and controls by the qPCR method which is also used for validation of microarray data (37). As mentioned before, miRNAs are a group of epigenetic modifications which are linked to the environment, so different environments and populations may affect on the expression pattern of miR-18b. On the other hand, using more samples and applying qPCR method which is more sensitive than microarray method, can be considered for the contradictory results of this study compared with microarray results.

\section{Acknowledgments}

This study was accomplished at the biology 
department of University of Isfahan, and was financially supported by the Graduate Office of the University of Isfahan.

\section{Authors' contributions}

AZ experimental design, collection and assembly of data, data analysis, interpretation and manuscript writing. HZ conception and design of the study, data analysis, interpretation, manuscript writing and final approval of the manuscript.

All authors contributed to and approved the manuscript

\section{Conflict of interest}

The authors declare no conflict of interest.

\section{References}

1. Groop L, Pociot F. Genetics of diabetes-Are we missing the genes or the disease? Mol Cell Endocrinol. 2014; 382(1):726-39. PMID: 23587769

2. Stankov K, Benc D, Draskovic D. Genetic and epigenetic factors in etiology of diabetes mellitus type 1. Pediatrics. 2013; 132(6):1112-22. PMID: 24190679

3. Atkinson MA, Eisenbarth GS, Michels AW. Type 1 diabetes. Lancet. 2014; 383(9911):69-82. PMID: 23890997

4. Onkamo P, Väänänen S, Karvonen M, Tuomilehto J. Worldwide increase in incidence of Type I diabetes-the analysis of the data on published incidence trends. Diabetologia. 1999; 42(12):1395-403. PMID: 10651256

5. Patterson CC, Dahlquist GG, Gyürüs E, Green A, Soltész G, Group ES. Incidence trends for childhood type 1 diabetes in Europe during 1989-2003 and predicted new cases 2005-20: a multicentre prospective registration study. Lancet. 2009; 373(9680):2027-33. PMID: 19481249

6. Polychronakos C, Li Q. Understanding type 1 diabetes through genetics: advances and prospects. Nat Rev Genet. 2011; 12(11):781-92. PMID: 22005987

7. Xie Z, Chang C, Zhou Z. Molecular mechanisms in autoimmune type 1 diabetes: a critical review. Clin Rev Allergy Immunol. 2014; 47(2):174-92. PMID: 24752371

8. Steck AK, Rewers MJ. Genetics of type 1 diabetes. Clin Chem. 2011; 57(2):176-85. PMID: 21205883

9. Wu Y-L, Ding Y-P, Gao J, Tanaka Y, Zhang W. Risk factors and primary prevention trials for type 1 diabetes. Int J Biol Sci. 2013; 9(7):666-79. PMID: 23904791

10. Morran MP, Vonberg A, Khadra A, Pietropaolo M. Immunogenetics of type 1 diabetes mellitus. Molecular aspects of medicine. $2015 ; 42: 42-60$.

11. Lehuen A, Diana J, Zaccone P, Cooke A. Immune cell crosstalk in type 1 diabetes. Nat Rev Immunol. 2010; 10(7):50113. PMID: 20577267

12. Kumar P, Subramaniyam G. Molecular underpinnings of Th17 immune-regulation and their implications in autoimmune diabetes. Cytokine. 2015; 71(2):366-76. PMID: 25510901
13. He L, Hannon GJ. MicroRNAs: small RNAs with a big role in gene regulation. Nature Reviews Genetics. 2004; 5(7):522-31.

14. Rauniyar VK, Wu W, Yang H. MicroRNAs in autoimmune disease. Auto Immun Highlights. 2011; 2(2):59-65. PMID: 26000120

15. Singh RP, Massachi I, Manickavel S, Singh S, Rao NP, Hasan $\mathrm{S}$, et al. The role of miRNA in inflammation and autoimmunity. Autoimmun Rev. 2013; 12(12):1160-5. PMID: 23860189

16. Hezova R, Slaby O, Faltejskova P, Mikulkova Z, Buresova I, Raja KM, et al. microRNA-342, microRNA-191 and microRNA510 are differentially expressed in $\mathrm{T}$ regulatory cells of type 1 diabetic patients. Cell Immunol. 2010; 260(2):70-4. PMID: 19954774

17. Sebastiani G, Grieco FA, Spagnuolo I, Galleri L, Cataldo D, Dotta F. Increased expression of microRNA miR-326 in type 1 diabetic patients with ongoing islet autoimmunity. Diabetes Metab Res Rev. 2011; 27(8):862-6. PMID: 22069274

18. Sebastiani G, Vendrame F, Dotta F, editors. MicroRNAs as new tools for exploring type 1 diabetes: relevance for immunomodulation and transplantation therapy. Transplant Proc. 2011; 43(1): 330-2. PMID: 21335216.

19. Salas-Pérez F, Codner E, Valencia E, Pizarro C, Carrasco E, Pérez-Bravo F. MicroRNAs miR-21a and miR-93 are down regulated in peripheral blood mononuclear cells (PBMCs) from patients with type 1 diabetes. Immunobiology. 2013; 218(5):733-7. PMID: 22999472

20. Takahashi P, Xavier DJ, Evangelista AF, Manoel-Caetano FS, Macedo C, Collares CV, et al. MicroRNA expression profiling and functional annotation analysis of their targets in patients with type 1 diabetes mellitus. Gene. 2014; 539(2):213-23. PMID: 24530307

21. Dweep H, Gretz N. miRWalk2. 0: a comprehensive atlas of microRNA-target interactions. Nat Methods. 2015; 12(8):697. PMID: 26226356

22. Dweep H, Sticht C, Pandey P, Gretz N. miRWalk-database: prediction of possible miRNA binding sites by "walking" the genes of three genomes. J Biomed Inform. 2011; 44(5):839-47. PMID: 21605702

23. Pfaffl MW, Horgan GW, Dempfle L. Relative expression software tool (RESTC) for group-wise comparison and statistical analysis of relative expression results in real-time PCR. Nucleic Acids Res. 2002; 30(9):e36. PMID: 11972351

24. Van Belle TL, Coppieters KT, Von Herrath MG. Type 1 diabetes: etiology, immunology, and therapeutic strategies. Physiol Rev. 2011; 91(1):79-118. PMID: 21248163

25. Waterhouse $\mathrm{P}$, Penninger JM, Timms E, Wakeham A. Lymphoproliferative disorders with early lethality in mice deficient in Ctla-4. Science. 1995; 270(5238):985-8. PMID: 7481803

26. Littman DR, Rudensky AY. Th17 and regulatory T cells in mediating and restraining inflammation. Cell. 2010; 140(6):84558. PMID: 20303875

27. Wing K, Onishi Y, Prieto-Martin P, Yamaguchi T, Miyara M, Fehervari Z, et al. CTLA-4 control over Foxp3+ regulatory $\mathrm{T}$ cell function. Science. 2008; 322(5899):271-5. PMID: 18845758

28. de Jong V, Zaldumbide A, van der Slik A, Persengiev S, Roep B, Koeleman B. Post-transcriptional control of candidate risk 
genes for type 1 diabetes by rare genetic variants. Genes Immun. 2013; 14(1):58-61. PMID: 22932817

29. Javierre BM, Hernando H, Ballestar E. Environmental triggers and epigenetic deregulation in autoimmune disease. Discov Med. 2011; 12(67):535-45. PMID: 22204770

30. Lu Q. The critical importance of epigenetics in autoimmunity. $\mathbf{J}$ Autoimmun. 2013; 41:1-5. PMID: 23375849

31. Brooks WH, Le Dantec C, Pers J-O, Youinou P, Renaudineau Y. Epigenetics and autoimmunity. J Autoimmun. 2010; 34(3):J207-J19. PMID: 20053532

32. Ceribelli A, Satoh M, Chan EK. MicroRNAs and autoimmunity. Curr Opin Immunol. 2012; 24(6):686-91. PMID: 22902047

33. Pauley KM, Cha S, Chan EK. MicroRNA in autoimmunity and autoimmune diseases. J Autoimmun. 2009; 32(3):189-94. PMID: 19303254
34. Baumjohann D, Ansel KM. MicroRNA-mediated regulation of $\mathrm{T}$ helper cell differentiation and plasticity. Nat Rev Immunolog 2013; 13(9):666-78. PMID: 23907446

35. Mogilyansky E, Rigoutsos I. The miR-17/92 cluster: a comprehensive update on its genomics, genetics, functions and increasingly important and numerous roles in health and disease. Cell Death Differ. 2013; 20(12):1603-14. PMID: 24212931

36. Otaegui D, Baranzini SE, Armañanzas R, Calvo B, MuñozCulla M, Khankhanian P, et al. Differential micro RNA expression in PBMC from multiple sclerosis patients. PloS One. 2009; 4(7):e6309. PMID: 19617918

37. Valasek MA, Repa JJ. The power of real-time PCR. Adv Physiol Educ. 2005; 29(3):151-9. PMID: 16109794 